\title{
A 21-year-old male with atraumatic right hip pain: diagnosis and discussion
}

\author{
Leon Sergot $^{1} \cdot$ Sian Davies ${ }^{1} \cdot$ Paul Davison ${ }^{1} \cdot$ Julian Chakraverty $^{1}$
}

Published online: 3 June 2020

(C) ISS 2020

\section{Test yourself: question}

A 21-year-old male with atraumatic right hip pain

\section{History}

A 21-year-old male initially presented to his General Practitioner with atraumatic right hip and thigh pain. He was subsequently referred for MRI of the hip/pelvis by an orthopaedic surgeon.

\section{Test yourself: answer}

A 21-year-old male with atraumatic right hip pain: diagnosis and discussion

\section{Diagnosis}

Subperiosteal haematoma of the left iliac bone

\section{Discussion}

Pelvic MRI revealed no right hip abnormality but an incidental lesion of the contralateral iliac bone: a superficial, partially cystic, lenticular lesion arising from the pelvic side of the left

The case presentation can be found at https://oi.org/10.1007/s00256020-03460-6

Leon Sergot

leon.sergot@doctors.org.uk

1 Department of Radiology, University Hospitals Bristol NHS Trust, Marlborough Street, Bristol BS1 3NU, UK iliac blade, underlying the iliacus muscle (Figs. 1, 2, 3, 4 and 5). There is a sclerotic border, no soft tissue component nor deep extension and no underlying bone marrow oedema. The sclerotic border and cystic component are appreciable on the radiograph (Fig. 6) but easily overlooked given the presence of overlying bowel gas.

These appearances are consistent with a subperiosteal haematoma of the left iliac bone. The smooth-edged lentiform morphology strongly implies a subperiosteal location, as demonstrated in other anatomical locations such as the calvarium [1], caused by blood products expanding into a non-extendable space. In this case, the contralateral location to that of the symptoms suggests chronicity. This is corroborated by imaging characteristics suggestive of chronic blood products: the central component is low/isointense on $\mathrm{T} 1$ and high on $\mathrm{T} 2$ and peripheral component low signal on both. Here, the low intensity peripheral component is more likely explained by peripheral ossification, a well-recognized process in haematoma evolution. Old, subsequently ossified subperiosteal haematomata have been described to demonstrate a specific sign coined the 'ghost cortex' [2]: On CT and MRI, the ossified haematoma can be seen overlying but not obscuring the native (or 'ghost') cortex - best demonstrated on Fig. 2.

More acute haematomata may demonstrate less ossification, and signal characteristics may also differ corresponding to the age of the blood products. Early phase lesions demonstrate callus formation in the absence of a fracture; it matures with ossification if the haematoma fails to resorb spontaneously [3, 4], as illustrated in this case. The loose attachment of the periosteum in adolescents results in susceptibility of traumatic detachment and injury to nutrient vessels [2, 5] resulting in haematoma formation. Differential diagnoses include surface bone lesions such as bone cysts. The non-medullary location would be atypical for a unicameral bone cyst and lack of fluidfluid levels inconsistent with aneurysmal bone cysts. The MRI appearances are similar to another rare entity, the periosteal/ subperiosteal ganglion; however, these are typically found in the lower limb long bones [6]. In the context of trauma, an 
intramuscular haematoma should also be considered; however, the location underlying the iliacus muscle and sparing of the psoas tendon and lentiform morphology favours a subperiosteal haematoma [2,5]. Significantly, the specific 'ghost cortex' sign helps clinch the diagnosis [2]. Importantly, the lack of aggressive periosteal reaction, soft tissue component or cortical destruction implies a non-aggressive aetiology.

Subperiosteal haematomata themselves are rare in the iliac bone but have been described. Where in the older population they are often found incidentally, in adolescents they present with pain and gait disturbance following trauma $[2,7]$ and have also been diagnosed in the setting of coagulation abnormalities [8]. It is postulated that pain and gait abnormalities arise from compression of the adjacent femoral nerve $[2,7,8]$. In this context, evacuation is indicated. Conservative management is sufficient in static lesions with no associated neurology [7].

\section{References}

1. Higashi R, Hayashi Y, Watanabe T, Hirota Y, Park CH, Hamada J. Huge recurrent subperiosteal hematoma in a 9-year-old boy: case report with special reference to pathologic and anatomic studies. Childs Nerv Syst. 2008;24:403-5.

2. Guillin R, Moser T, Koob M, et al. Subperiosteal hematoma of the iliac bone: imaging features of acute and chronic stages with emphasis on pathophysiology. Skelet Radiol. 2012;41(6):667-75.

3. Seeger L, Yao L, Eckardt J. Surface lesions of bone. Radiology. 1998;206:17-33.

4. Lee SH, Yun SJ. Iliac subperiosteal hematoma with ossification in a 15-year-old boy. J Pediatr. 2017;190:285-285.e1.

5. Gitto S, Pescatori L, Aliprandi A, Sconfienza L. Subperiosteal hematoma of the ilium: an unusual complication of acetabular fracture. Eurorad. 2016. Case 14182.

6. Reghunath A, Mittal K, Khanna G, Anil V. Tibial periosteal ganglion cyst: the ganglion in disguise. Ind $\mathrm{J}$ Radiology and Imaging. 2017;27:105-9.

7. Yumoto T, Joko R, Yamakawa Y, Yamada T, Naito H, Nakao A. Subperiosteal hematoma of the iliac bone: an unusual cause of acute hip pain after a fall. Am J Case Rep. 2018;19:1083-6.

8. Weiss JM, Tolo V. Femoral nerve palsy following iliacus haematoma. Orthopedics. 2008;31(2):178.

Publisher's note Springer Nature remains neutral with regard to jurisdictional claims in published maps and institutional affiliations. 